\title{
Going beyond 10-meter, Gbit/s underwater optical wireless communication links based on visible lasers
}

\author{
Chao Shen, ${ }^{1}$ Yujian Guo, ${ }^{1}$ Xiaobin Sun, ${ }^{1}$ Guangyu Liu, ${ }^{1}$ Kang-Ting Ho, ${ }^{1}$ Tien Khee Ng, ${ }^{1}$ Mohamed-Slim Alouini, ${ }^{2}$ \\ and Boon S. Ooi ${ }^{1, *}$ \\ ${ }^{1}$ Photonics Laboratory, King Abdullah University of Science and Technology (KAUST), Thuwal 23955, Saudi Arabia \\ ${ }^{2}$ Communication Theory Laboratory, King Abdullah University of Science and Technology (KAUST), Thuwal 23955, Saudi \\ Arabia \\ *E-mail address: boon.ooi@kaust.edu.sa
}

\begin{abstract}
Laser diode based visible light communication link with data rate of 2.2 Gbps over a 12-meter underwater channel is reported using non-return-to-zero on-off keying modulation scheme. The underwater optical wireless communication link beyond 10-meter can be achieved using visible lasers.
\end{abstract}

Keywords—diode laser; InGaN laser; visible lasers; underwater optical wireless communication; visible light communication

\section{INTRODUCTION}

GaN-based laser diodes (LDs) and superluminescent diodes (SLDs) have exhibited a number of advantages over lightemitting diodes (LEDs) in the blue-green-red color regime for high transmission speed, large bandwidth indoor and outdoor optical wireless communications applications [1-5]. LD based integrated photonic systems have recently demonstrated for such applications [6-8]. Using the on-off-keying (OOK) modulation scheme, a LD based free-space visible light communication (VLC) link has been developed with a high data rates of $>2$ Gbps, serving the functionalities of lighting and data communication [1]. Moreover, the LD based underwater wireless optical communication (UWOC) system has recently been proposed to meet the large data rate demands in underwater video streaming, sea floor monitoring, and offshore oil exploration applications $[9,10]$. However, the channel length for the demonstrated LD based UWOC link is relatively short (few meters) so far [10-12]. Achieving a UWOC channel of $>10 \mathrm{~m}$ will significantly enhance flexibility and pervasiveness in outdoor and field applications. In this paper, we report on the implementation of a high-speed LD based VLC link offering a data rate up to 2.2 Gbps over a 12-meter-long UWOC channel using the least complex and the most cost-effective OOK modulation technique. We demonstrate that the corresponding bit-error rates (BER) surpassed the forward error correction (FEC) limit of $3.8 \times 10^{-3}$ using a low-cost $\mathrm{Si}$ photodetector (PD) as the receiver.

\section{EXPERIMENTAL DETAILS}

A TO-can packaged single-mode InGaN/GaN quantum well based blue emitting LD was used as the transmitter in this study. Figure 1 presents the optical power - current - voltage (L-I-V) relations of the LD at room temperature. A threshold current of $25 \mathrm{~mA}$ and an optical power of $58 \mathrm{~mW}$ at $80 \mathrm{~mA}$ injection current have been measured. The emission spectra of the LD were measured using Ocean Optics HR 4000 high-resolution

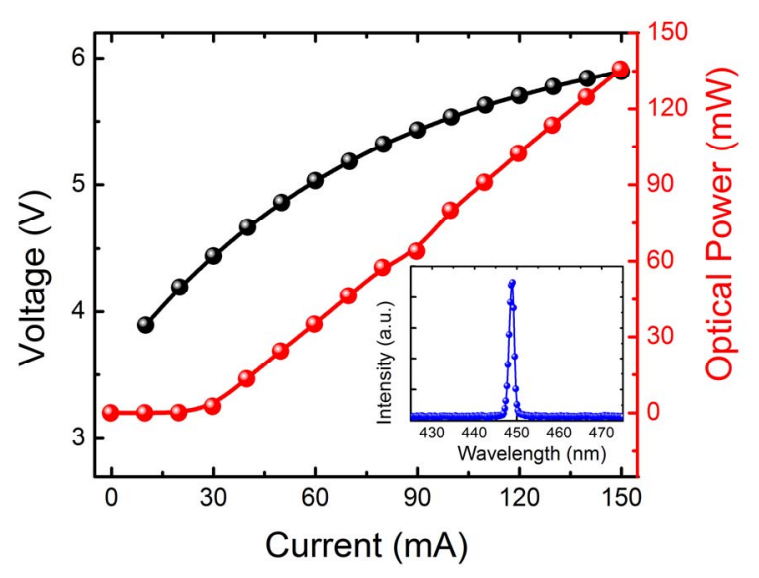

Fig. 1. L-I-V characteristics of the blue InGaN-based laser diode. Inset: emission spectra of the LD at $80 \mathrm{~mA}$.

spectrometer (inset of Fig. 1), showing a peak emission wavelength of $\sim 450 \mathrm{~nm}$.

Figure 2 presents the schematic of the experimental setup for laser based UWOC set-up using the non-return-to-zero, on-offkeying (NRZ-OOK) modulation scheme. The LD is driven using a Keithley 2400 source meter as the direct current (DC) source. The pattern generator in the Agilent N4903B J-BERT is used to generate the pseudorandom binary sequence (PRBS) $2^{10}$ 1 pattern, and the data stream is amplified by the $28-\mathrm{dB}$ driver amplifier before connecting to the TEK PSPL 5580 broadband bias-tee. A plano-convex lens is used at the receiver end to focus the optical signal into the Menlo Systems APD210 Si avalanche PD. An Agilent $86100 \mathrm{C}$ digital communications analyzer

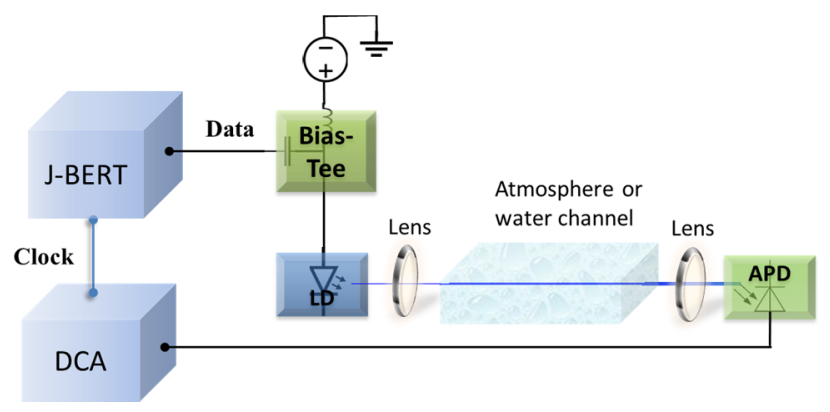

Fig. 2. Schematic of the measurement setup. 
(DCA) was used to collect the eye diagram and the error performance is obtained using the J-BERT.

\section{RESULTS AND DISCUSSIONS}

(a)

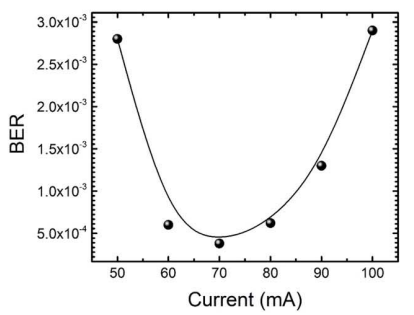

(b)

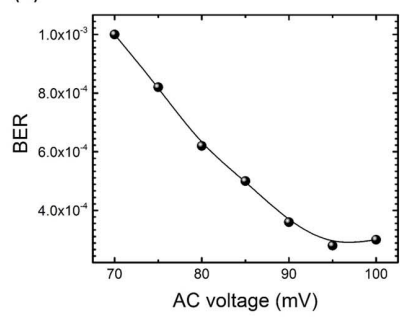

Fig.3. Optimization of the DC (a) and AC (b) bias point of the laser diode. Lowest BER is achieved at a DC driving current of $70 \mathrm{~mA}$ and $\mathrm{AC}$ modulation voltage of $95 \mathrm{mV}$.

A throughput optimization of the operation point was performed before the experiment by adjusting the injection current and the modulation signal amplitude to bias the LD. For the NRZ-OOK modulation of the LD, changes of BER at different operating currents [Fig. 3(a)] and at a different peakto-peak voltage from the pattern generator [Fig. 3(b)] were measured at a data rate of 2 Gbps. Under low driving voltage, the relatively low signal-to-noise ratio may lead to a high BER. However, if the driving voltage is too high, the signal may also be affected by the transient heating. The minimum BER is found
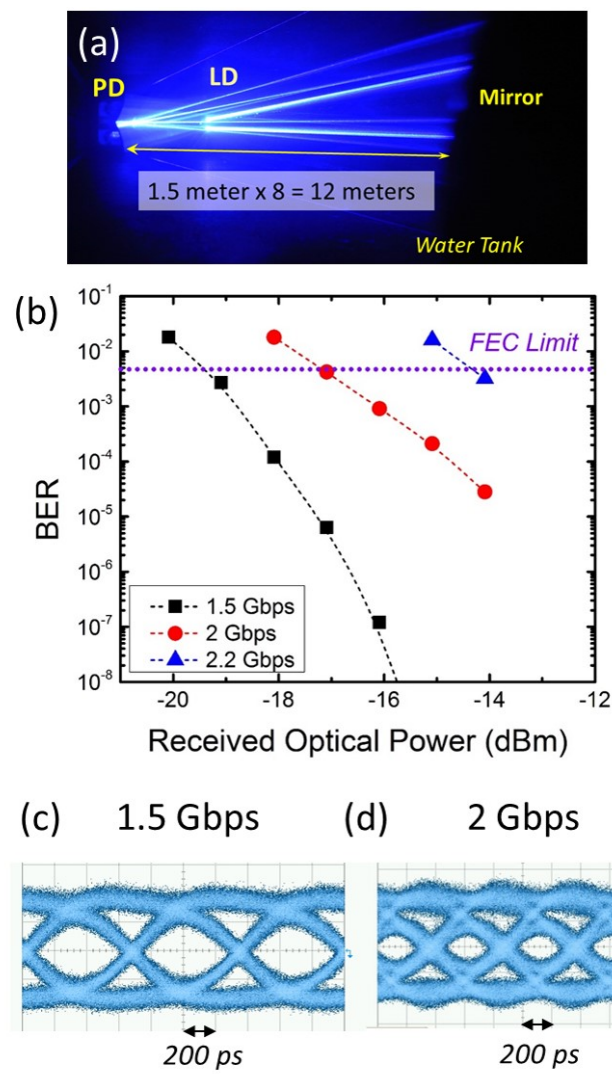

(d) 2 Gbps

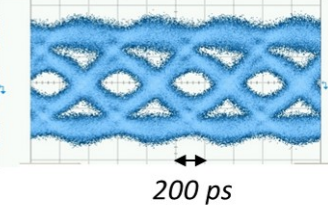

Fig. 4. (a) Photo of the 12-meter UWOC link. (b) Plot of BER as a function of received optical power at data rates of $1.5 \mathrm{Gbps}, 2 \mathrm{Gbps}$ and 2.2 Gbps. The eye diagrams at (c)1.5 Gbps and (d) 2 Gbps. at the driving current of $70 \mathrm{~mA}$ and encoding amplitude of 95 $\mathrm{mV}$, where the LD shows the optimized performance.

Figure 4(a) shows the implementation of the LD-based VLC system for a 12-meter water channel. Two mirrors were placed on each side of the 1.5-meter long water tank to reflect the laser beam to achieve 12-meter long beam transmission path. The water tank was filled with tap water. The measured BER performance as a function of the received optical power at the date rates of $1.5 \mathrm{Gbps}, 2 \mathrm{Gbps}$, and 2.2 Gbps is shown in Fig. 4(b). A data rate of $2.2 \mathrm{Gbps}$ has been achieved at a received optical power of $-14 \mathrm{dBm}$. A minimum received optical power of $\sim-19 \mathrm{dBm}$ and $-16 \mathrm{dBm}$ is required to achieve a below-FEClimit BER for $1.5 \mathrm{Gbps}$ and $2 \mathrm{Gbps}$, respectively. The corresponding eye diagrams are shown in Fig. 4(c) and Fig. 4(d). To investigate the further extending of the transmission distance, we calculated the received optical power through the underwater channel according to the Beer-Lambert law:

$$
I=I_{0} e^{-c x}
$$

For LD-based UWOC system implemented in the clear ocean, an attenuation coefficient, c, of $0.056 \mathrm{~m}^{-1}$ is expected. As a result, the received optical power after a 40-meter transmission channel is estimated to be $\sim-19 \mathrm{dBm}$. According to the measured BER in Fig. 4(b), the presented LD-based UWOC system is expected to achieve 1.5 Gbps over a 40 -meter UWOC link.

\section{CONCLUSIONS}

In summary, we have experimentally demonstrated LDbased UWOC link beyond 10-meter transmission distance, which can be further extended to 40-meter. The long range UWOC links are practical for eventual field deployment.

\section{ACKNOWLEDGMENT}

We acknowledge the financial support from King Abdulaziz City for Science and Technology (KACST), Grant No. KACST TIC R2-FP-008, and KACST-KAUST-UCSB solid state lighting program (SSLP). This work is partially supported by baseline funding, BAS/1/1614-01-01, from the King Abdullah University of Science and Technology (KAUST).

\section{REFERENCES}

[1] C. Lee, C. Shen, H. M. Oubei, M. Cantore, B. Janjua, T. K. Ng, et al., "2 Gbit/s data transmission from an unfiltered laser-based phosphorconverted white lighting communication system," Optics Express, 23, 29779-29787 (2015).

[2] F. Zafar, M. Bakaul, and R. Parthiban, "Laser-Diode-Based Visible Light Communication: Toward Gigabit Class Communication," IEEE Communications Magazine, 55, 144-151 (2017).

[3] C. Shen, T. K. Ng, J. T. Leonard, A. Pourhashemi, S. Nakamura, S. P. DenBaars, et al., "High-brightness semipolar (20) 21 ) blue $\mathrm{InGaN} / \mathrm{GaN}$ superluminescent diodes for droop-free solid-state lighting and visiblelight communications," Optics Letters, 41, 2608-2611 (2016).

[4] C. Shen, C. Lee, T. K. Ng, S. Nakamura, J. S. Speck, S. P. DenBaars, et al., "High-speed 405-nm superluminescent diode (SLD) with 807-MHz modulation bandwidth," Optics Express, 24, 20281-20286 (2016).

[5] C. Shen, J. T. Leonard, E. C. Young, T. K. Ng, S. P. DenBaars, J. S. Speck, et al., "GHz modulation bandwidth from single-longitudinal mode 
violet-blue VCSEL using nonpolar InGaN/GaN QWs," Conference on Lasers and Electro-Optics (CLEO), p. STh1L.2 (2016).

[6] C. Shen, T. K. Ng, J. T. Leonard, A. Pourhashemi, H. M. Oubei, M. S. Alias, et al., "High-Modulation-Efficiency, Integrated Waveguide Modulator-Laser Diode at 448 nm," ACS Photonics, 3, 262-268 (2016).

[7] C. Shen, C. Lee, E. Stegenburgs, J. H. Lerma, T. K. Ng, S. Nakamura, et al., "Semipolar III-nitride quantum well waveguide photodetector integrated with laser diode for on-chip photonic system," Applied Physics Express, 10, 042201 (2017).

[8] C. Shen, C. Lee, T. K. Ng, S. Nakamura, J. S. Speck, S. P. DenBaars, et al., "High gain semiconductor optical amplifier - Laser diode at visible wavelength," 2016 IEEE International Electron Devices Meeting (IEDM), pp. 22.4.1-22.4.4 (2016)
[9] C. Shen, Y. Guo, H. M. Oubei, T. K. Ng, G. Liu, K.-H. Park, et al., "20meter underwater wireless optical communication link with $1.5 \mathrm{Gbps}$ data rate," Optics Express, 24, 25502-25509 (2016).

[10] J. Xu, Y. H. Song, X. Y. Yu, A. B. Lin, M. W. Kong, J. Han, et al., "Underwater wireless transmission of high-speed QAM-OFDM signals using a compact red-light laser," Optics Express, 24, 8097-8109 (2016).

[11] H. M. Oubei, C. Li, K.-H. Park, T. K. Ng, M.-S. Alouini, and B. S. Ooi, "2.3 Gbit/s underwater wireless optical communications using directly modulated $520 \mathrm{~nm}$ laser diode," Optics Express, 23, $20743-20748$ (2015).

[12] K. Nakamura, I. Mizukoshi, and M. Hanawa, "Optical wireless transmission of $405 \mathrm{~nm}, 1.45 \mathrm{Gbit} / \mathrm{s}$ optical IM/DD-OFDM signals through a $4.8 \mathrm{~m}$ underwater channel," Optics Express, 23, 1558-1566 (2015). 